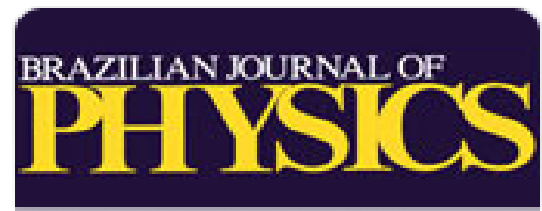

Brazilian Journal of Physics

ISSN: 0103-9733

luizno.bjp@gmail.com

Sociedade Brasileira de Física

Brasil

Hernández de la Luz, A. D.; Rodríguez Moreno, M. A.

Casimir Force Between Two Spatially Dispersive Dielectric Parallel Slabs

Brazilian Journal of Physics, vol. 41, núm. 4-6, 2011, pp. 216-222

Sociedade Brasileira de Física

Sâo Paulo, Brasil

Available in: http://www.redalyc.org/articulo.oa?id=46421512002

How to cite

Complete issue

- More information about this article

Journal's homepage in redalyc.org

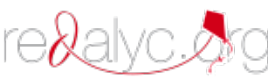

Scientific Information System Network of Scientific Journals from Latin America, the Caribbean, Spain and Portugal Non-profit academic project, developed under the open access initiative 


\title{
Casimir Force Between Two Spatially Dispersive Dielectric Parallel Slabs
}

\author{
A. D. Hernández de la Luz • M. A. Rodríguez Moreno
}

Received: 29 October 2010 / Published online: 20 September 2011

(C) Sociedade Brasileira de Física 2011

\begin{abstract}
We investigate the Casimir force $F$ between two parallel spatially dispersive semiconductor slabs, whose dielectric response function accounts for nonlocal effects. Nonlocal effects are induced by the presence of excitonic transitions in the semiconductor slabs for which our studies consider the $A_{n=1}$ exciton in $\mathrm{CdS}$ and the $\mathrm{Z}_{3} 1 s$ one in $\mathrm{CuCl}$. In order to explore the nonlocal effects in the Casimir force, we first calculate the $S$ and $P$ polarized reflection coefficients of the excitonic slabs then we use them in the functional form of $\mathrm{F}$. The slabs are considered as homogeneous, and nonhomogeneous media where the latter is a periodic system having a unit cell with period $d$. We present numerical calculations of $F$ as a function of the vacuum gap of width $L$ between the slabs, different slab thicknesses, and periods. Comparisons between numerical results obtained by using a nonlocal and a local theory show that the nonlocal effects are more significant at short separations of the slabs. $F$ suffers a small decrease as a consequence of the energy absorption induced by the excitons.
\end{abstract}

Keywords Casimir force $\cdot$ Exciton $\cdot$ Nonlocal effects

\section{Introduction}

Since the Casimir effect was discovered by Casimir [1-3], it has attracted a great attention. Originally, this is a purely quantum mechanical effect which is manifested as an

A. D. H. de la $\operatorname{Luz}(\bowtie) \cdot$ M. A. R. Moreno

CIDS-ICUAP Benemérita Universidad Autónoma de Puebla,

Apdo. Postal 1651,

Puebla, Puebla 72000, Mexico

e-mail: jaherlu@siu.buap.mx attractive force between non-charged materials. In the framework of quantum field theory, these interactions can be described through the alteration induced by the presence of material boundaries to the zero-point electromagnetic energy which pervades all space. Nowadays, several methods for calculating the Casimir forces have been developed such that it is possible to reach an arbitrary accuracy for any number of objects, arbitrary shapes, susceptibility functions, and separations [4, 5]. The development of methods to measure Casimir forces with high precision and the advent of nano- and micromechanical devices where Casimir forces play an important role have detonated the interest to study these forces both theoretically and experimentally [6, 7]. Recent papers report important advances in this direction [8].

The Casimir force being so important in nano- and microtechnological applications, a great effort has been made to extend theories such that real materials can be included in the analysis of this phenomenon. So, during the last few years significant progress has been obtained not only in the measurement of the Casimir force but also in the development of new calculational methods applicable to nontrivial geometries taking into account real material properties of the interacting bodies [8-11].

New geometrical arrangements and materials have been reported in recent articles to compute the Casimir force, resulting in great interest of those related to the optical properties of the media [12]. The force has been estimated as not only using a simple material such as a metal and a dielectric but also complex materials such as metamaterials, ferromagnetic and diamagnetic materials, and polymers [13-16].

It is found that the Casimir force is dependent on the optical properties of the system and its geometric config- 
uration. Most of the studies consider a local theory for the dielectric response function for which $\varepsilon=\varepsilon(\omega)$. The numerous applications of the Casimir force and the extensive experimental work impose several demands on the accuracy of theoretical predictions, among them those which correspond to optical characterization.

Because of the Casimir force having a great sensitivity to the optical response of the system, it results in having a great importance to include a nonlocal theory in the treatment of the optical response. The latter is relevant when we consider semiconductor materials; there, the presence of excitonic transitions which induce nonlocal effects and consequently significant variations on the reflectivity spectrum is possible, thus inducing changes in the Casimir force. A good approach which considers the nonlocal effects or spatial dispersion effects gives that the dielectric response function is dependent on the frequency and the wave vector, in this case $\varepsilon=$ $\varepsilon(\omega, \vec{q})$ [17]. In previous reports, spatial dispersion effects have been considered in the Casimir force in metals [18-20]. In the case of dielectrics, as a first approximation, calculations were done for the force in the one-dimensional approach considering a geometry of two parallel slabs [21, 22].

Even though spatial dispersion effects are only important in the vicinity of the excitonic transitions, they should be incorporated in the calculations of the Casimir force to make a correct description of the optical response. In this work, we present a study of the Casimir force between parallel slabs made up of excitonic semiconductors whose dielectric function is nonlocal. We investigate the force accounting for the $A_{n=1}$ exciton in $\mathrm{CdS}$ and the $\mathrm{Z}_{3} 1 \mathrm{~s}$ one in $\mathrm{CuCl}$. We introduce the excitonic effects by a frequency and wave vectordependent dielectric response $\varepsilon(\omega, \vec{q})$. To calculate the Casimir force, we use the formula of $F$ which was obtained using the Green's functions formalism that allows a direct calculation if the slab reflectivities are known. The outline of this paper is as follows: we describe the formalism in Section 2; Section 3 is devoted in discussing the results and making conclusions.

\section{Formalism}

Let us consider two parallel excitonic semiconductor slabs as depicted in Fig. 1. The coordinate system is shown; the $x y$ plane is parallel to the slab surfaces where the $z$-axis is perpendicular to them. We need the exact calculus of their reflection coefficients in the inner boundaries due to interaction with the vacuum electromagnetic modes in between the slabs. For solving this problem, we assume

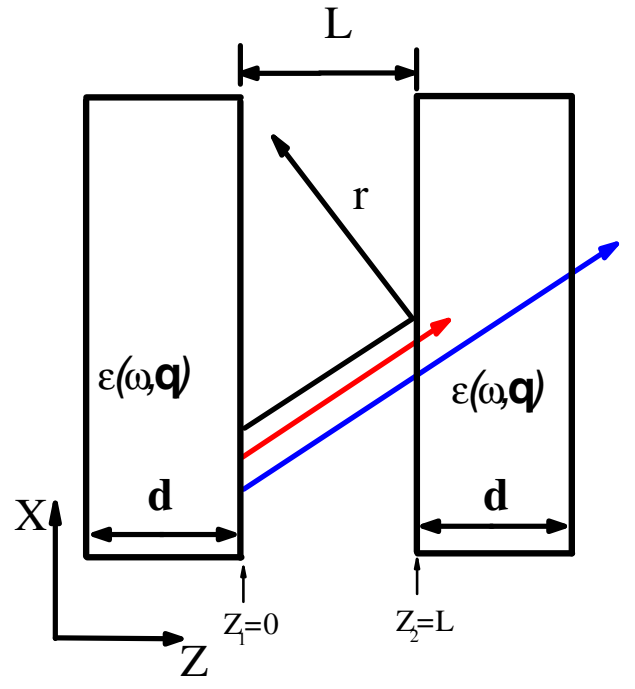

Fig. 1 (Color online) Schematic representation of the two nonlocal semiconductor slabs which are parallel to the $x y$ plane and apart from a distance $L$ having inner boundaries at $z_{1}$ and $z_{2}$; each one with a thickness $d$ and a dielectric response function $\varepsilon(\omega, \vec{q})$. Interaction of the electromagnetic radiation confined in the vacuum cavity of width $L$ is quantified by the reflection coefficient $r$

that the slabs $a=1,2$ are parallel to the $x y$ plane within free space and separated by a distance $L$ along the $z$-direction, with inner boundaries at $z_{1}$ and $z_{2}$ as shown in the figure. We consider that the slabs are translational invariant, nonchiral and isotropic within the $x y$ plane but otherwise they may be arbitrary. They are identical to each other and have nonlocal dielectric responses and, in principle, with a finite thickness. The description of the electromagnetic fields within the vacuum taking into account the excitonic modes generated in the excitonic semiconductor slabs has been widely studied by using the $Z$ surface impedance approach of any planar surface [17]. Remarkable aspects from this topic is that $Z$ is defined as the ratio of the complex electric and magnetic tangential field components at the surface.

All information about matter-radiation interaction within the slabs are included in their suitable reflection amplitudes $r_{a}^{\alpha}(a=1,2 ; \alpha=\mathrm{s} ; p)$ for both $S$ and $P$ polarization which are determined by the surface impedance $Z_{a}^{\alpha}$ given by

$r_{a}^{\alpha}=\frac{Z_{a}^{\alpha}-Z_{0}^{\alpha}}{Z_{a}^{\alpha}+Z_{0}^{\alpha}}$

$Z_{a}^{\alpha}$ is defined as the ratio $\frac{E_{a}}{H_{a}}$ of the components $E_{a}$ and $H_{a}$ of the electric and magnetic fields evaluated at the $a$ th interface for outgoing boundary conditions [23]. $Z_{0}^{\alpha}$ is the surface impedance of vacuum for which $Z_{0}^{S}=\frac{q}{k}$ and $Z_{0}^{P}=$ $\frac{k}{p}$ where $\vec{q}=(Q, 0, \pm k)$ is the vacuum wave vector. The 
nonlocality will be included in the study of the optical properties of the excitonic semiconductor slabs by the presence of excitonic transitions, namely, the $A_{n=1}$ exciton in $\mathrm{CdS}$ [17] and also the $Z_{3} 1 s$ exciton in $\mathrm{CuCl}$ [24] as materials of study. We employ a nonlocal dielectric function for CdS given by

$\varepsilon(\omega, \vec{q})=\varepsilon_{0}+\frac{\omega_{p}^{2}}{\omega_{T}^{2}+D \vec{q}^{2}-\omega^{2}-i v \omega}$

and for $\mathrm{CuCl}$,

$\varepsilon(\omega, \vec{q})=\varepsilon_{\infty}\left(1-\frac{\omega_{L}^{2}-\omega_{T}^{2}}{\omega^{2}-\omega_{T}^{2}-D \vec{q}^{2}+i v \omega}\right)$

In both equations, $\omega$ is the frequency of light, $\vec{q}$ is the wave vector and $D \vec{q}^{2}=\left(\frac{h \omega_{T}}{M}\right) \vec{q}^{2}$ describe the influence of the nonlocality with $M$ the exciton's mass. Additionally, $\omega_{T}$ determines the energy $\hbar \omega_{T}$ required to create an exciton in each material.

For oblique incidence of light, there are transverse modes excited in the slabs which satisfy the dispersion relation $\varepsilon(\omega, \vec{q})=\frac{q^{2} c^{2}}{\omega^{2}}$ and longitudinal modes with the dispersion relation $\varepsilon(\omega, \vec{q})=0$. Through (2) in these dispersion relations we obtain four transverse waves of which two travel in the forward and two in the backward directions, besides, two longitudinal modes one which travels in the forward and another in the backward directions. When $P$ polarized light is incident onto a slab both transverse and longitudinal modes are excited within the slab. On the other hand, if $S$ polarized light is considered, only transverse modes are excited in the slabs. $P$ and $S$ polarizations are uncoupled, therefore, we deal with them independently, first, with $S$ and second, with $P$ polarized waves to determine the reflection coefficients.

When $S$ polarized light interacts with a semiconductor slab, there are four transverse modes propagating towards the right and left with wave vectors $(Q, 0$, $\left.\pm q_{j}\right)$ where $\pm q_{j}(j=1,2)$ are the solutions of the transverse wave dispersion relation. By assuming that the electromagnetic field is given by a superposition of these four waves, then anywhere the field inside the slab is determined by four independent field quantities at one point, namely, the tangential components of the electric and magnetic field, $E_{y}(z)$ and $H_{x}(z)$, the excitonic polarization vector $P_{y}(z)$ and its normal derivative $\partial_{z} P_{y}(z)$. The field components are written in terms of the amplitudes $E_{n}^{ \pm}$of the transverse modes that travel to the right $(+)$ and to the left $(-)$. To solve the amplitudes $E_{n}^{ \pm}$ we use the transfer matrix formalism and we construct a $2 \times 2$ transfer matrix which relates the fields at one interface $z_{i}^{l}$ to the fields in another one at $z_{i}^{r}$, where $l$ is the left interface and $r$ is the right one [17]. According to this method, these fields may be written in a general matrix form as

$$
\left(\begin{array}{l}
F \\
P
\end{array}\right)_{z}=G\left(\begin{array}{l}
A_{1} \\
A_{2}
\end{array}\right)_{z}
$$

where $F=\left(\begin{array}{c}E_{y} \\ H_{x}\end{array}\right), P=\left(\begin{array}{c}P_{y} \\ \partial_{z} P_{x}\end{array}\right), A_{n}=\left(\begin{array}{c}E_{n}^{+} e^{i q_{n} z} \\ E_{n}^{-} e^{-i q_{n} z}\end{array}\right)$.

The fields at the left $z_{i}^{l}$ and the right $z_{i}^{r}=z_{i}^{l}+d$ boundaries of the slab satisfy the equation

$$
\left(\begin{array}{l}
F \\
P
\end{array}\right)_{z_{i}^{r}}=M\left(\begin{array}{l}
F \\
P
\end{array}\right)_{z_{i}^{l}}
$$

The transfer matrix $M$ relates the fields at the $z_{i}^{r}$, the right boundary of the slab, to the fields at $z_{i}^{l}$, its left boundary. Here, $M=G T(d) G^{-1}$ is a $4 \times 4$ transfer matrix, $d$ is the thickness of the slab, $T(d)=\operatorname{diag}\left(e^{i q_{1} d}, e^{-i q_{2} d}, e^{i q_{2} d}, e^{-i q_{2} d}\right)$ is a diagonal matrix. The fields $E_{y}, H_{x}, P_{y}, \partial_{z} P_{y}$ in the bulk are independent, but at surface they are related to each other by the additional boundary conditions (ABC) and the boundary conditions of electromagnetic origin. In this report, we take as the (ABC) those which consider $\left[\alpha \vec{P}+\partial_{n} \vec{P}\right]_{S}=0$ where $\partial_{n} \vec{P}$ is an outward normal derivative and $\alpha$ is a parameter. By applying the (ABC) and the Maxwell boundary conditions it is possible to collapse the dimension of the $4 \times 4$ transfer matrix to $2 \times 2$ one, such that we obtain $\left(\begin{array}{c}E_{y} \\ H_{x}\end{array}\right)_{z_{i}^{r}}=M_{t}\left(\begin{array}{c}E_{y} \\ H_{x}\end{array}\right)_{z_{i}^{l}}$ where the $M_{t}$ matrix includes information of the multiple electromagnetic modes which exist in the semiconductor slab with frequencies near the excitonic transition ones due to spatial dispersion generated by the excitons. Accordingly, the use of this equation allows one to calculate the surface impedance $Z_{a}^{S}\left(z_{a}\right)=\frac{E_{y}\left(z_{a}\right)}{H_{x}\left(z_{a}\right)}$ as well as the reflection coefficients from (1).

With regard to the $P$ polarized waves, six independent normal modes propagate within the slabs, from them four are normal modes with wave vectors $\left(Q, 0, \pm q_{j}\right)$ where $\pm q_{\mathrm{j}}(j=1,2)$ which in turn are the solutions of the transverse wave dispersion relation and the others are two longitudinal modes with wave vectors $\left(Q, 0, \pm q_{3}\right)$ being $q_{3}$ the solution of the longitudinal dispersion relation $\varepsilon\left(\omega, Q, 0, q_{3}\right)=0$. Therefore, six independent fields must be considered everywhere inside the slabs, namely, the electric and magnetic fields $E_{x}, H_{y}$, the excitonic polarization fields, $P_{x}, P_{z}$, and their normal derivative $\partial_{z} P_{x}$ and $\partial_{z} P_{z}$. 
Following the formalism developed in Ref. [17], it may be possible to write the fields in a matrix form

$$
\left(\begin{array}{l}
F \\
P \\
\partial_{z} P
\end{array}\right)_{z_{i}^{r}}=M_{p}\left(\begin{array}{l}
F \\
P \\
\partial_{z} P
\end{array}\right)_{z_{i}^{l}}
$$

where $F=\left(\begin{array}{c}E_{x} \\ H_{y}\end{array}\right), P=\left(\begin{array}{c}P_{x} \\ P_{z}\end{array}\right), \partial_{z} P=\left(\begin{array}{c}\partial_{z} P_{x} \\ \partial_{z} P_{z}\end{array}\right)$ and the transfer matrix $M_{p}=G_{p} T(d) G_{p}^{-1}$ is a $6 \times 6$ transfer matrix and $G_{p}$ the matrix related to the six amplitudes $E_{n}^{ \pm}$of the fields. Again as above, by applying the (ABC) and the typical Maxwell boundary conditions it is possible to collapse the $6 \times 6 M_{p}$ matrix to $2 \times 2 M_{t}$ one such that $\left(\begin{array}{c}E_{x} \\ H_{y}\end{array}\right)_{z_{i}^{r}}=M_{t}\left(\begin{array}{c}E_{x} \\ H_{y}\end{array}\right)_{z_{i}^{l}}$ and we calculate the surface impedance $Z_{a}^{P}\left(z_{a}\right)=\frac{E_{x}\left(z_{a}\right)}{H_{y}\left(z_{a}\right)}$ and the reflection coefficients $r_{a}^{p}$.

Now, we consider the slabs with a periodic dielectric structure and define a unit cell as two consecutive layers of insulator and excitonic semiconductor, where the former has a dielectric function $\varepsilon_{I}$ and thickness $d_{I}$ and the latter $\varepsilon_{S}$ and $d_{S}$, respectively. To calculate the reflection coefficients for this periodic dielectric structure, we consider this system as a semi-infinite superlattice made up of alternating insulator layers and excitonic semiconductor ones. The excitonic semiconductor layers have a wave vector and frequency dependent dielectric function $\varepsilon_{s}(\omega, \vec{q})$ as given by (3) while the insulator layers are dispersionless arbitrary dielectrics. In accordance with Ref. [17] in the framework of the matrix transfer formalism, the surface impedance for the semi-infinite superlattices is

$Z_{a}^{\alpha}=-\frac{M_{(a) 12}^{\alpha}}{M_{(a) 11}^{\alpha}-e^{i p d}}=-\frac{M_{(a) 22}^{\alpha}-e^{i p d}}{M_{(a) 21}^{\alpha}}$

Here $M_{(a) m n}^{\alpha}$ are the components of the $2 \times 2$ transfer matrix for one full superlattice period $d=d_{I}+d_{S}$, for which $M_{(a)}^{\alpha}=M_{(a) I}^{\alpha} M_{(a) S}^{\alpha}$ where $M_{(a) I}^{\alpha}$ is the $2 \times 2$ transfer matrix for the insulator layer and $M_{(a) S}^{\alpha}$ is the $2 \times 2$ collapsed one for the excitonic semiconductor layer, besides, $p$ is Bloch's wave vector. Equation 7 allows us to calculate the reflection coefficients for each polarization $(\alpha=S, P)$ and slab $(a=1$, 2) by (1).

For the calculation of the Casimir force $F$ between two parallel slabs, we follow the formalism developed in Ref. [23] which considers two arbitrary slabs being parallel to the $x y$ plane in free space and separated by a distance $L$ along the $z$-direction. Taking into account both the $S$ and $P$ polarization of light with non normal incidence onto inner boundaries of the slabs, it is obtained the total force per unit area between the slabs which is given by

$$
\frac{F(L)}{A}=\frac{\hbar c}{2 \pi^{2}} \int_{0}^{\infty} \mathrm{d} Q Q \int_{q \geq 0} \mathrm{~d} k k^{2} f \operatorname{Re}\left\{\frac{r_{1}^{S} r_{2}^{S} e^{2 i k L}}{1-r_{1}^{S} r_{2}^{S} e^{2 i k L}}+\frac{r_{1}^{P} r_{2}^{P} e^{2 i k L}}{1-r_{1}^{P} r_{2}^{P} e^{2 i k L}}\right\}
$$

where $\vec{q}_{ \pm}=(Q, 0, \pm k)$ is the wave vector of the vacuum modes. This expression is applicable to our system; we can see that it depends on both the reflection coefficients and the slab separations.

\section{Results and Conclusions}

Along this section we explore the spatially dispersive effects on the Casimir force by computing this one using (8). The parameters used for the actual calculations are taken from the literature. On one hand, for the $A_{n=1}$ exciton, the parameters are [17]: $\varepsilon_{0}=9.1, \hbar \omega_{T}=2.55272 \mathrm{eV}$, $\omega_{p}=0.1151 \omega_{T}, D=5.3147 \times 10^{-6} c^{2}$ and $v=4.857 \times 10^{-5}$. On the other hand, for the $Z_{3} l s$ exciton [24], $M=2.3 m_{0}$, here $m_{0}$ is the free electron mass, the frequency for the exciton resonance $\hbar \omega_{T}=3.2022 \mathrm{eV}, \varepsilon_{\infty}=5.0$, the longitudinal-transverse splitting $\hbar \omega_{L T} \equiv \hbar\left(\omega_{L}-\omega_{T}\right)=$ $5.7 \mathrm{meV}$, in this case $v$ obeys the relation $v=v_{0}+$
$\gamma\left(\omega-\omega_{T}\right) \theta\left(\omega-\omega_{T}\right)$ where $\theta(x)$ is the Heaviside function and we take values $\hbar v_{0}=0.3 \mathrm{meV}$ and $\gamma=0.29$.

We use the Pekar boundary conditions in the (ABC) equation, in such case, we choose $\alpha \rightarrow \infty$. Two-dimensional numerical integration is carried out to determine the Casimir force taking into account the vacuum electromagnetic modes within the cavity of width $L$ which impinge onto the semiconductor slabs with wave vector $\vec{q}_{ \pm}=(Q, 0, \pm k)$ at non normal incidence and at zero temperature for which case $f=1 / 2$ in (8). The latter is a good approximation since the excitons in semiconductors are more likely to be existing at very low temperatures. The Casimir force results are presented in terms of the relative Casimir force $F / F_{i}$ in such a way that the ideal case (force between two parallel perfect conductors in which case $F_{i}=-\hbar \pi^{2} \mathrm{c} / 240 L^{4}$ ) corresponds to $F / F_{i}=1$.

As a first case of study, we present the behavior of the Casimir force as a function of slab separations $L$ considering various thicknesses $d$ including the limit case 
of two half-spaces as well as the $\mathrm{CuCl}$ as material forming the homogeneous slabs. Figure 2 shows that the relative force decreases as the separation between slabs increases, but it is augmented as the slab thickness is greater being in the limit case for half-spaces where the force reaches the highest intensity in such case it depends on separation $L$ as in the ideal case. Because the relative force is always positive then the Casimir force between slabs is attractive. Transparency of radiation through the slabs is more drastic in as much as we have thinner ones so, the density of electromagnetic energy confined in the vacuum cavity is diminished, this causes that the force between slabs decreases. This is true because the Casimir force is strongly dependent on electromagnetic energy in the vacuum cavity and this energy is reduced as the slab thickness is thinner and in this situation we have more transparency consequently the force has a less strength. Conversely, there is more electromagnetic energy whether the slab thickness is greater because the transparency is lesser then the force increases until it reaches a top value corresponding to the case of half-spaces configuration. In order to stand out the nonolocal effects in the Casimir force, we make comparisons between local $F_{l}$ and nonlocal $F_{n l}$ forces by using $\Delta=\frac{F_{l}-F_{n l}}{F_{l}}$. We point out that $F_{l}$ is obtained from the local theory approach where the slabs have a dielectric response function as $\varepsilon_{S}=\varepsilon_{S}(\omega)$ which means that we have the limit $q \rightarrow 0$ in (2) and (3), it implies that there are only transverse modes excited within the semiconductor slabs. Figure 3 shows the behavior of these comparisons. It is known that spatial dispersive

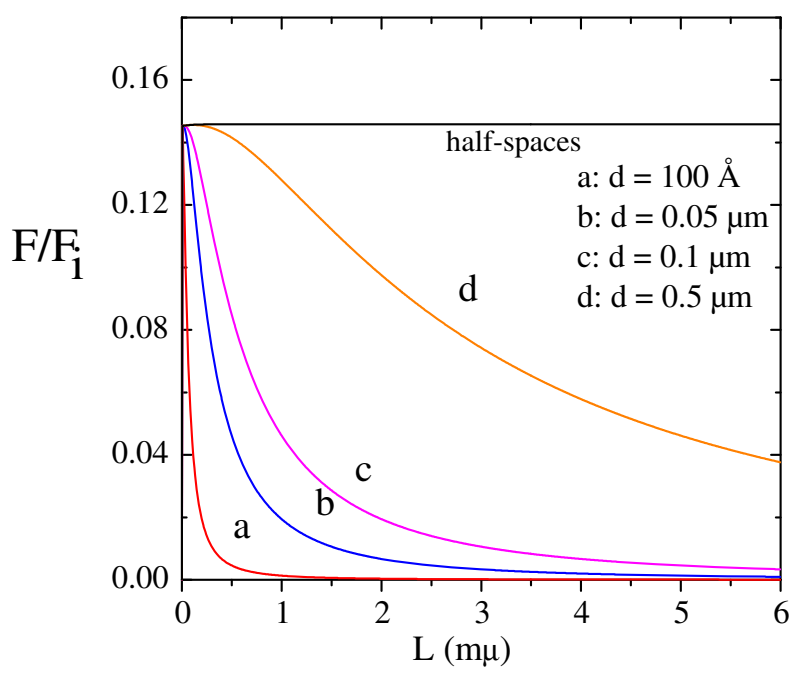

Fig. 2 (Color online) Relative Casimir force considering slabs formed by $\mathrm{CuCl}$ as a function of their thickness $d$ and separation $L$. The thinner the slabs are, the weaker force intensity will be, particularly at small separations $L$. The highest intensity of the force is found in the limit of half-spaces when $d \rightarrow \infty$

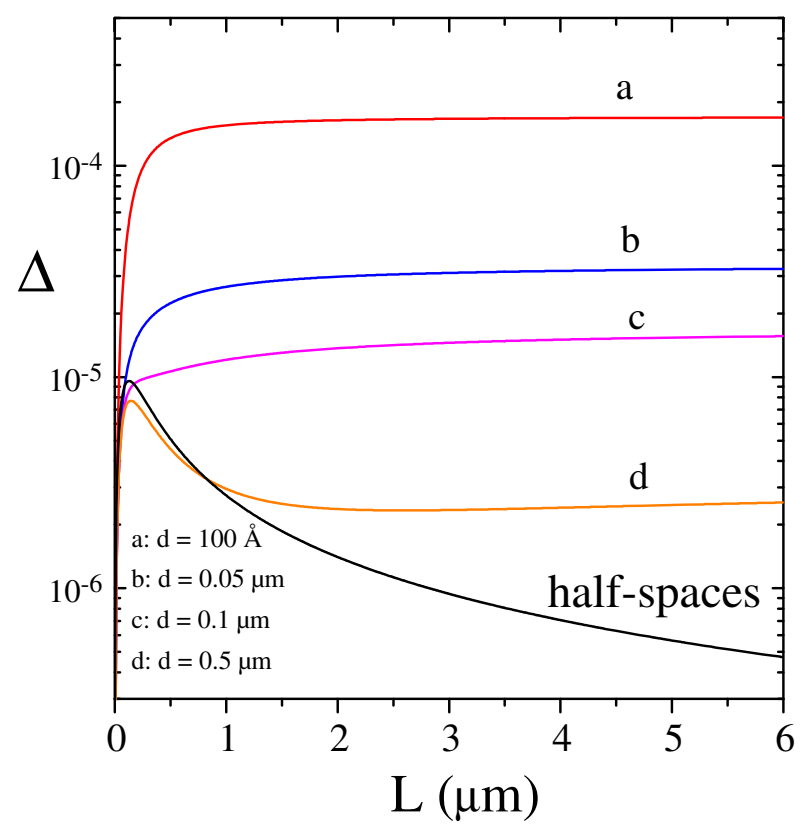

Fig. 3 (Color online) Comparisons between both local and nonlocal Casimir forces. In a few percentage, the local Casimir force is reduced in intensity due to the influence of the spatially dispersive effects generated by excitonic transitions excited within the semiconductor

effects generated by excitons are remarkable only in the neighborhood of the excitonic transition frequencies, then the reflectivities and the Casimir force will be affected significantly only in this frequency region which is around $\omega_{T}$ resonant frequency.

The manifold electromagnetic waves that may exist within the spatially dispersive slabs, longitudinal and transverse modes, induce all the structure of the nonlocal reflectivities, when these ones are compared with the reflectivities obtained by local theory approach, it is found that the additional longitudinal modes produce differences between them around $\omega_{T}$, see Fig. 4. The additional energy taken from the fields to induce the excitonic polarization effects, roughly speaking, may decrease the reflectivity of the system, consequently the nonlocal Casimir force may diminish its intensity. In Fig. 3, we realize that the relative differences are small being more meaningful for very thin slabs. Because of the short range of frequencies where nonlocal effects are remarkable it entails that the Casimir force undergoes a little reduction on its strength. Similar qualitative behavior is found when we use the $A_{n=1}$ exciton in CdS to study the Casimir Force (results not reported here). Referring back to Fig. 4, there we depict the reflectivities of the $\mathrm{CuCl}$ slabs considering only the $\mathrm{P}$ polarized light impinging at $\theta=60^{\circ}$ onto the slabs in order to stress the nonlocal effects. We observe as an important feature that nonlocal effects exist significantly only in the 


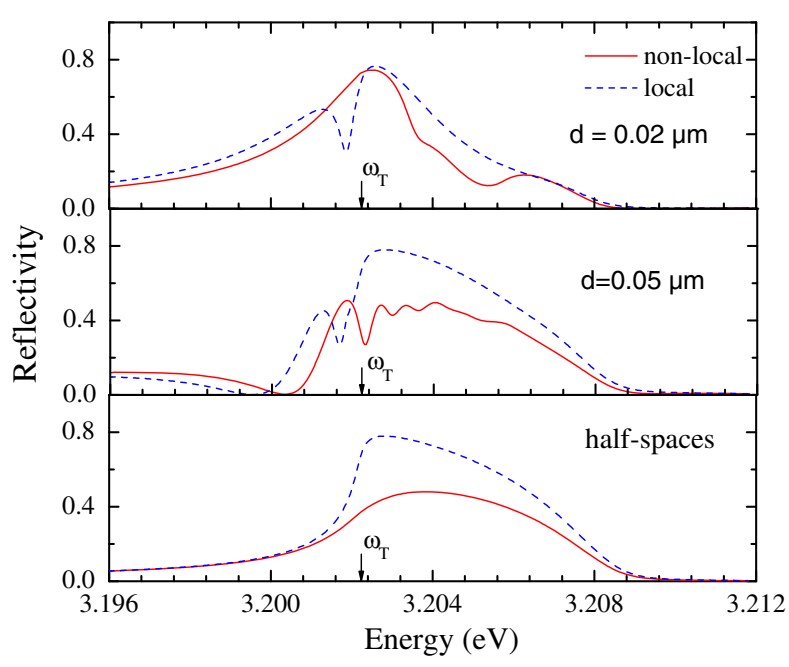

Fig. 4 (Color online) Reflectivity curves for $\mathrm{CuCl}$ slabs considering $P$ polarized light at $\theta=60^{\circ}$. The presence of the transverse and longitudinal electromagnetic modes in the nonlocal reflectivity spectrum dictate the behavior of this curve. These electromagnetic modes make the difference when we compare both the local reflectivity and nonlocal one

vicinity of $\omega_{T}$, outside this region neither local reflectivity nor nonlocal reflectivity contributes to the Casimir force.

In Fig. 5, we depict the nonlocal Casimir force evaluated using as a material of study CdS. For this new case, the slabs are made of semi-infinite superlattices which are built by alternating dispersionless layers with

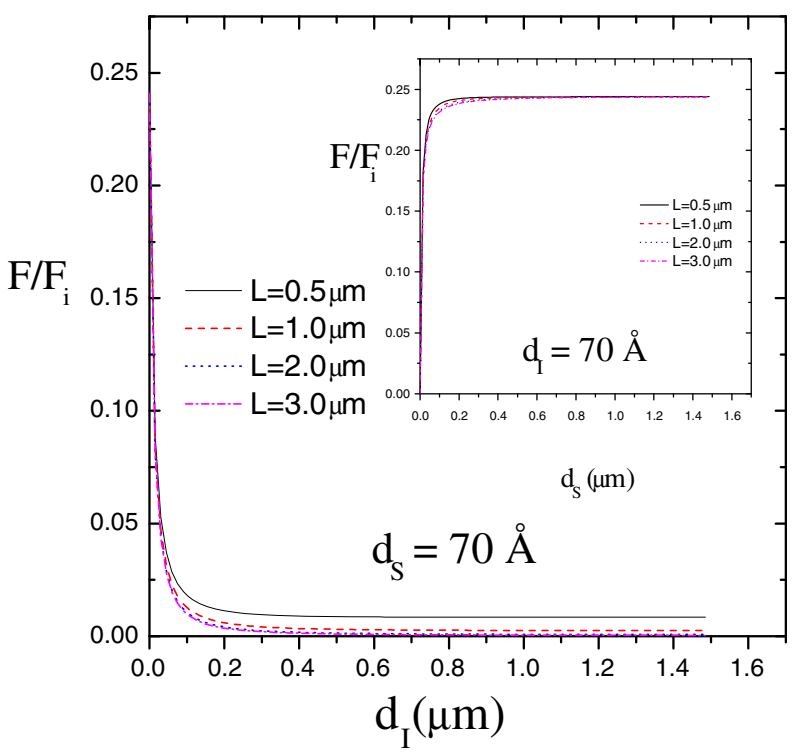

Fig. 5 (Color online) Casimir force corresponding to two slabs made up semi-infinite superlattices. The unit cell of the superlattice has an insulate layer with period $d_{I}$ and dielectric response function $\varepsilon_{I}=1$ and an excitonic semiconductor layer with one $d_{S}$ and one $\varepsilon_{S}(\omega, \vec{q})$ dielectric function $\varepsilon_{I}=1$ and thickness $d_{I}$ and excitonic semiconductor ones with dielectric response function given by (2) and thickness $d_{S}$. The period of the superlattice is $d=d_{I}+d_{S}$. We plot in this figure the Casimir force varying the period of superlattice while the separation between slabs is held constant. As the period of the superlattice increases by varying the dispersionless layer thickness $d_{I}$ the force changes drastically from a maximum value reached at small periods to lower values which approximate to a minimum constant value. Also, as the separation between slabs increases, the force decreases to zero. The inset in the same figure, points out that the force behaves contrary when the dispersionless thickness is kept constant and we vary the excitonic semiconductor one $d_{S}$. It is clear that the force reaches quickly a maximum with no significant variations when both $L$ and $d_{S}$ increase. These results let us obtain a region of $d$ where the force varies sharply because it may change from upper (lower) to lower (upper) values by only increasing either $d_{I}$ (keeping $d_{S}$ fixed) or $d_{S}$ (keeping $d_{I}$ fixed). This behavior is attributable to both the influence of the geometric factor, variations of $d$, and the presence of the longitudinal and transverse electromagnetic modes within the excitonic layer whose coupling with the transverse modes within the dispersionless layer at the interface between each layer determines the reflectivities of this kind of systems. Although we do not present results in this report when comparisons are made between values of the force using the local and non local approach, it is worthwhile to stress that similar qualitative results are obtained as the ones presented above for the homogeneous slabs.

In summary, we have presented a study of the Casimir force considering the contribution of the spatially dispersive effects or nonlocal effects for a system of two semiconductor parallel slabs. By making comparisons between the Casimir force obtained in the framework of the local theory and the one obtained in the nonlocal approach we found that the presence of the longitudinal and transverse electromagnetic modes excited within the slabs account for the reduction of the Casimir force. These modes are due to the excitonic transitions of $A_{n=1}$ exciton in $\mathrm{CdS}$ and the $Z_{3} 1 s$ exciton in $\mathrm{CuCl}$. For the case of homogeneous slabs, the nonlocal effects induce variations in a few percentage on the force which are more meaningful for thin slabs, this result leads us to consider that the local theory is enough to study this system. Finally, for the case of nonhomogeneous slabs, we found that the strength of the Casimir force changes sharply when we increase the period of the periodic structure that makes up each slab. Besides the force is almost independent on the separations between slabs. 
Acknowledgments This work was partially supported by the CIDSICUAP and VIEP-BUAP projects.

\section{References}

1. H.B.G. Casimir, Proc. K. Akad. Wet. Amsterdam 51, 793 (1948)

2. H.B.G. Casimir, in Proc. of the Royal Netherlands Academy of Arts and Sciences, Amsterdam, 1997, vol 61, ed. by L.M. Schoonhoven

3. S.K. Lamoreaux, Rep. Prog. Phys. 68, 201 (2005)

4. S. Jamal Rahi, T. Emig, N. Graham, Phys. Rev. D 80, 085021 (2009)

5. G. Jordan Maclay, Phys. Rev. A 82, 032106 (2010)

6. S.K. Lamoreaux, Phys. Rev. Lett. 78, 5 (1997)

7. H.B. Chan, V.A. Aksyuk, R.N. Kleiman, D.J. Bishop, F. Capasso, Phys. Rev. Lett. 87, 211801 (2001)

8. G.L. Klimchitskaya, Rev. Mod. Phys. 81, 1821 (2009)

9. B. Geyer, G.L. Klimchitskaya, V.M. Mostepanenko, Phys. Rev. A 67, 062102 (2003)

10. M. Shaden, Phys. Rev. A 82, 022113 (2010)

11. S. Zaher, S. Jamal Rahi, T. Emig, R.L. Jaffe, Phys. Rev. A 81, 030502 (2010)
12. Y. Yang, R. Zeng, H. Chen, S. Zhu, M. Zubairy, Pys. Rev. A 81, 022114 (2010)

13. G. Metalidis, P. Bruno, Phys. Rev. A 81, 022123 (2010)

14. F. Kheirandish, M. Soltani, J. Sarabadani, Phys. Rev. A 81, $0522110(2010)$

15. M. Benhamou et al., EJTP 5(9), 141 (2008)

16. F.S.S. Rosa, D.A.R. Dalvit, P.W. Milonni, Phys. Rev. A. 78, 032117 (2008)

17. G.H. Cocoletzi, W.L. Mochán, Surf. Sci. 57, 1-58 (2005)

18. A.M. Contrera-Reyes, W.L. Mochán, Phys. Rev. A 7(2), 034102 (2005)

19. R. Esquivel-Sirvent, V.B. Svetovoy, Phys. Rev. A 69, 062102 (2004)

20. R. Esquivel-Sirvent, V.B. Svetovoy, Phys. Rev. B 69, 062102 (2005)

21. A.D. Hernández de la Luz, A.F. Alvarado, G.H. Cocoletzi, R. Esquivel-Sirvent, Solid State Commun. 132, 623-626 (2004)

22. A.D. Hernández de la Luz, M.A. Rodríguez Moreno, J. Olvera Hernández, G. Hernández Cocoletzi, Superficies y Vacío 19(2), 7 (2006)

23. R. Esquivel, C. Villareal, W.L. Mochán, Phys. Rev. A 6(8), 052103 (2003)

24. A. Silva-Castillo, F. Pérez-Rodríguez, J. Appl. Phys. 90, 3662 (2001) 